\title{
DNA methylation status of nuclear-encoded mitochondrial genes underlies the tissue-dependent mitochondrial functions
}

\author{
Masaki Takasugi ${ }^{1}$, Shintaro Yagi ${ }^{1}$, Keiji Hirabayashi ${ }^{1}$, Kunio Shiota ${ }^{1,2^{*}}$
}

\begin{abstract}
Background: Mitochondria are semi-autonomous, semi-self-replicating organelles harboring their own DNA (mitochondrial DNA, mtDNA), and their dysregulation is involved in the development of various diseases. While mtDNA does not generally undergo epigenetic modifications, almost all mitochondrial proteins are encoded by nuclear DNA. However, the epigenetic regulation of nuclear-encoded mitochondrial genes (nuclear mt genes) has not been comprehensively analyzed.

Results: We analyzed the DNA methylation status of 899 nuclear mt genes in the liver, brain, and heart tissues of mouse, and identified 636 nuclear mt genes carrying tissue-dependent and differentially methylated regions (T-DMRs). These nuclar $\mathrm{mt}$ genes are involved in various mitochondrial functions and they also include genes related to human diseases. T-DMRs regulate the expression of nuclear mt genes. Nuclear mt genes with tissuespecific hypomethylated T-DMRs were characterized by enrichment of the target genes of specific transcription factors such as FOXA2 in the liver, and CEBPA and STAT1 in the brain.
\end{abstract}

Conclusions: A substantial proportion of nuclear mt genes contained T-DMRs, and the DNA methylation status of numerous T-DMRs should underlie tissue-dependent mitochondrial functions.

\section{Background}

Mitochondrial dysfunction is a common cause of human diseases [1,2], and thus understanding the regulation of mitochondrial functions is critical. Mitochondria do not contain histones [3], and almost all mtDNA is unmethylated $[4,5]$, indicating that mtDNA is not epigenetically regulated. However, while mammalian mitochondria are estimated to consist of more than 1,500 proteins, only 13 proteins are encoded by mtDNA.

Methylation of nuclear DNA is a major component of epigenetic system in mammalian cells, and is involved in silencing of gene transcription and maintaining genomic stability [6,7]. Hypomethylation of regulatory regions is required to allow expression of genes [8,9]. Microarraybased DNA methylation analysis revealed the existence of thousands of tissue-dependent and differentially

\footnotetext{
* Correspondence: ashiota@mail.ecc.u-tokyo.ac.jp

'Laboratory of Cellular Biochemistry, Department of Animal Resource Science/Veterinary Medical Sciences, the University of Tolyo, Tokyo 113-8657, Japan

Full list of author information is available at the end of the article
}

methylated regions (T-DMRs) in the mouse and human genomes $[10,11]$. While the T-DMRs of some genes, such as Oct-4 and Nanog, are hypomethylated only in a few cells $[12,13]$, the methylation status of most T-DMRs is not specific, but common to certain cells or tissues $[11,14]$. Tissue-dependent methylation status of T-DMRs, including tissue-specific methylation status of T-DMRs, forms a distinctive DNA methylation profile for each cell type $[8,11,15]$.

A nuclear mt gene, Ant4, which encodes mitochondrial outer membrane protein, contains T-DMRs which is specifically hypomethylated in the testis $[16,17]$. Also, few dozens of nuclear mt genes in mice are hypomethylated in the liver relative to the cerebrum [11]. However, the presence of T-DMRs in nuclear mt genes has not been comprehensively analyzed; this is necessary for understanding the regulation of mitochondrial functions. In this study, we analyzed the DNA methylation of 899 nuclear $\mathrm{mt}$ genes in the liver, brain, and heart tissues of mouse; these tissues consume large amounts of energy and are highly susceptible to mitochondrial 
dysfunctions. Our results indicated that at least 636 nuclear $\mathrm{mt}$ genes, which account for $71 \%$ of the total investigated nuclear $\mathrm{mt}$ genes, contain T-DMRs in their transcription start site (TSS) flanking regions $(-7 \sim+3 \mathrm{~kb}$ of TSSs), and that the differential methylation status of these T-DMRs is associated with tissue-dependent mitochondrial functions.

\section{Results and Discussion}

Identification of T-DMRs in the TSS flanking regions of nuclear mt genes in the liver, brain, and heart tissues

To investigate the DNA methylation status of nuclear $\mathrm{mt}$ genes in the liver, brain (cerebrum), and heart tissues, we conducted pairwise tissue comparisons using model-based analysis of tiling-array (MAT) along with D-REAM analysis [11,18]. Differences in the DNA methylation status at $\mathrm{HpyCH} 4 \mathrm{IV}$ sites (ACGT sites) were exhibited as differences in MATscores of the probes corresponding to the selectively amplified fragments generated by digestion of unmethylated HpyCH4IV sites [11]. We identified tissue-dependent and differentially methylated HpyCH4IV sites within the $10 \mathrm{~kb}$-TSS flanking regions of 899 RefSeq genes that are known to encode mitochondrial proteins and are registered in the Mitop2 database as a reference set [19]. In each tissue comparison, HpyCH4IV sites with MATscores above a specific threshold value were identified as hypomethylated T-DMRs in that particular tissue. The lowest MATscore of HpyCH4IV sites whose hypomethylation were confirmed by combined bisulfite restriction analysis (COBRA) (Figure $1 \mathrm{~A}, \mathrm{~B}$ and Additional file 1,2), were considered as the threshold values of MATscore. COBRA was performed for HpyCH4IV sites whose MATscores were larger than 2 when one tissue was compared to the either of the other 2 tissues. We identified T-DMRs in the $10 \mathrm{~kb}$-TSS flanking regions of 636 nuclear mt genes (Figure $1 \mathrm{C}$ and Additional file 3), with 123,119, and 99 nuclear $\mathrm{mt}$ genes with T-DMRs specifically hypomethylated in the liver, brain, and heart tissues, respectively, when compared with the other 2 tissues (hereafter referred to as tissuehypo T-DMRs) (Figure 1C and Additional file 4).

Most functional categories of mitochondria classified by Mitop2 were found in nuclear $m$ t genes with T-DMRs and with hypo T-DMRs (Figure 1D). Overrepresentation and underrepresentation of nuclear $\mathrm{mt}$ genes with liver-hypo T-DMRs were found in the detoxification and mitochondrial ribosomal categories, respectively $(P<0.05$, Fisher's exact test; Figure $1 \mathrm{D}$ and Table 1). Detoxification is one of the functions of the liver. Nuclear $\mathrm{mt}$ genes with liver-hypo T-DMRs also included the genes related to liver-specific functions, namely, Otc and Lrpprc (Table 1). OTC functions in the urea cycle in the liver [20]. LRPPRC is a binding partner of PPARGC1A, and functions in hepatic gluconeogenesis [21]. Nuclear mt genes with T-DMRs contained a number of genes involved in various types of mitochondrial regulation, such as the regulation of protein sorting, mitochondrial morphology, and translation of mtDNA-encoded proteins (Table 1).

Human homologs of mouse nuclear mt genes possessing T-DMRs are related to various human diseases. For example, mutations of Lrpprc, Ndufs 4 , and $N d u f s 8$, the genes with liver-, brain-, and heart-hypo T-DMRs, respectively, are associated with Leigh's disease [21-23]. In addition to mutations, overexpression of some nuclear mt genes with liver-hypo T-DMRs in non-liver tissues are involved in the human diseases. For example, overexpression of Acsl5 and Tgm2 are found in the human glioma and brain of Huntington's disease and are suggested to be involved in pathogenesis $[24,25]$.

\section{Correlation between the DNA methylation status of T-DMRs and nuclear $\mathrm{mt}$ gene expression}

Next, we examined the correlation between the DNA methylation status of nuclear $\mathrm{mt}$ genes and their expression using microarray data downloaded from Gene Expression Omnibus (Figure 2A and Additional file 5). The liver-hypo T-DMRs positively correlated with the liver vs. cerebral cortex and liver vs. heart expression ratios (Figure 2A). In the brain and heart, only the hypo T-DMRs located downstream of TSSs positively correlated with the ratio of the expression in hypomethylated tissue vs. other tissue (Figure 2A and Additional file 5). On the other hand, negative correlation was observed between the upstream heart-hypo T-DMRs and the heart vs. cerebral cortex expression ratio, although the average expression levels were greater in the heart (Figure 2A). Thus, the downstream hypo T-DMRs correlated with the expression of the nuclear $\mathrm{mt}$ genes in all tissues examined. The correlation between T-DMRs around TSSs and tissue-specific gene expression coincided with that in the previous reports $[11,15,26,27]$. We confirmed the tissue-dependent gene expression of Otc, Acsl6, and Ndufs4, the genes carrying downstream hypo T-DMRs for liver, brain, and heart, respectively, by using real-time PCR (Figure 2B).

\section{Concentration of liver-hypo T-DMRs in the downstream regions of nuclear $\mathrm{mt}$ genes}

In addition to the highest correlation of downstream liver-hypo T-DMRs with nuclear mt gene expression (Figure 2A and Additional file 5), a significantly larger proportion of the nuclear $\mathrm{mt}$ genes with liver-hypo T-DMRs contained these hypo T-DMRs in their downstream regions as compared to the nuclear $\mathrm{mt}$ genes with brain- and heart-hypo T-DMRs $\left(P<5 \times 10^{-3}\right.$, Fisher's exact test; Figure $3 \mathrm{~A})$. Furthermore, in genes with 


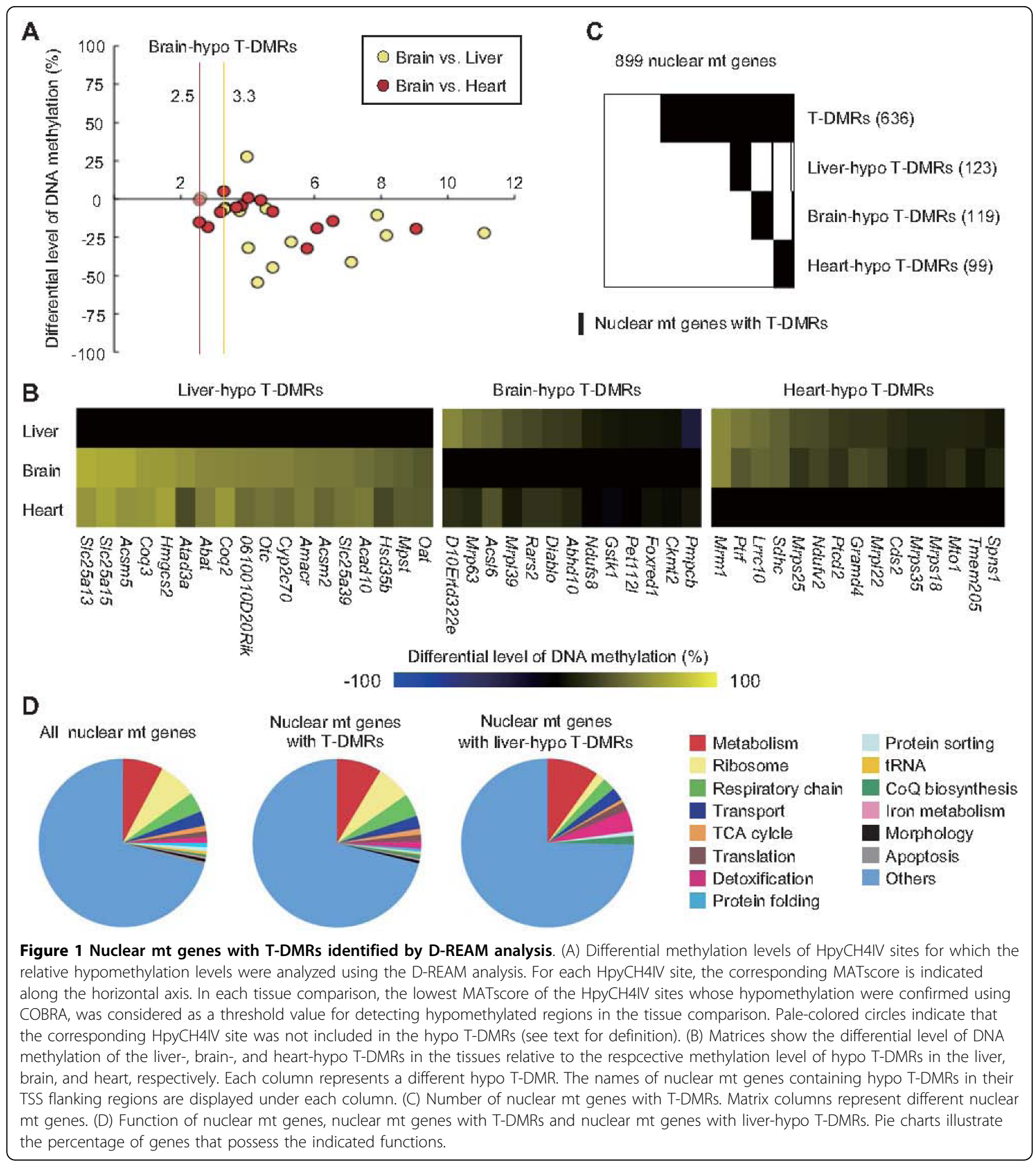

downstream liver-hypo T-DMRs, the nuclear mt genes were highly enriched (Figure 3B). Liver-hypo T-DMRs were especially enriched within regions of $+1 \sim+2 \mathrm{~kb}$ of TSSs of nuclear mt genes (Figure 3C). These results indicate that regulation of nuclear $\mathrm{mt}$ genes is especially dependent on DNA methylation in the liver.
Overrepresented transcriptional regulatory motifs in nuclear mt genes with liver- and brain-hypo T-DMRs We next investigated whether T-DMRs of nuclear $\mathrm{mt}$ genes are associated with any transcriptional regulatory motifs. FOXA2 is a transcription factor that activates the genes involved in mitochondrial $\beta$-oxidation and the 
Table 1 Nuclear mt genes with hypo T-DMRs

\begin{tabular}{lccc}
\hline & Liver-hypo T-DMRs & Brain-hypo T-DMRs & Heart-hypo T-DMRs \\
\hline Various mitochondrial functions & & & \\
\hline Metabolism & Abat, Acs15, Oat, etc & Dmgdh, Mthfd1, Star, etc & Cpt16, Efta, Pdk4, etc \\
\hline Respiratory chain & Atp50, Ndufb2, etc & Atp51, Cox6, Ndufs8, etc & Atp5l, Ndufs4, Sdhc, etc \\
\hline TCA cycle & Dlst & Aco2, Dlst & Ogdh \\
\hline Detoxification & Gsr, Hagh, Mgst1, Tst & Gstk1 & \\
\hline Tissue-specific mitochondrial functions & Otc, Lrpprc & Tomm7 & \\
\hline Protein sorting & Immp1l & Mfn1, Prelid1 & \\
\hline Regulation of mitochondrial morphology & & & Mrrf, Tufm \\
\hline Regulation of mtDNA & Peo1 & Mtif2, Mtif3 & \\
\hline Translation of mtDNA-encoded proteins & Lrpprc & \\
\hline
\end{tabular}

regulation of lipid metabolism, ketogenesis, and insulin sensitivity in the mouse liver $[28,29]$. We analyzed the enrichment of genes containing FOXA2-binding sites within an extended gene region (encompassing $10 \mathrm{~kb}$ upstream of TSS and $1 \mathrm{~kb}$ downstream of the 3' UTR) using known genome-wide FOXA2-binding sites in the mouse liver obtained by ChIP-sequencing [30]. We found that the targets of FOXA2 were enriched 1.6-fold in nuclear $\mathrm{mt}$ genes with liver-hypo T-DMRs relative to all nuclear mt genes $\left(P<5 \times 10^{-5}\right.$, Fisher's exact test). Sixty-four out of 123 nuclear mt genes with liver-hypo T-DMRs were the targets of FOXA2 (Additional file 6). This is contrast to the previous report, which indicated enrichment of target genes of HNF1 and/or HNF4 in hypomethylated genes in the mouse liver [11].

We further analyzed the enrichment of specific regulatory motifs in nuclear mt genes with hypo T-DMRs by using the oPOSSUM program [31]. We used all nuclear $\mathrm{mt}$ genes as a background gene set, and analyzed both $5 \mathrm{~kb}$ upstream and downstream of TSSs of the genes with hypo T-DMRs. The enrichment of the genes with FOXA2-binding sites in their downstream region was observed in the nuclear $\mathrm{mt}$ genes with liver-hypo T-DMRs. (Table 2 and Additional file 7). It has been reported that FOXA2-binding at distal downstream region of TSS of CEBPA gene functions as an enhancer in humans [32]. Hypomethylation of downstream T-DMRs may enhance transcription by permitting transcription factors access to target genes. We also found that genes with NFYA-binding sites in their downstream regions were enriched among the nuclear mt genes with liver-hypo T-DMRs, and the genes with CEBPA- and STAT1-binding sites in their upstream regions were enriched among the nuclear mt genes with brain-hypo T-DMRs (Table 2 and Additional file 7). These results indicate that T-DMRs are associated with specific transcription factors in a tissue-dependent manner. CEBPA and STAT1 are reported to be involved in cortical neurogenesis [33] and inflammatory responses in the brain
[34], respectively. It has been reported that CEBPA-null mice showed decreased nuclear $\mathrm{mt}$ gene expression in brown adipose tissue [35]. STAT1 also regulates nuclear $\mathrm{mt}$ gene expression in T cells in response to IFN- $\gamma$ [36]. Hypomethylation of hypo T-DMRs of targets of these transcription factors may permit transcription factors access to the target genes, and hypermethylation of these T-DMRs may be essential for secured repression of the corresponding genes in other tissues.

\section{Conclusion}

At least $71 \%$ of investigated nuclear mt genes contains T-DMRs, and the methylation status of T-DMRs correlated with tissue-dependent expression of dozens of nuclear $\mathrm{mt}$ genes. Considering that there are at least 200 different cell types in the mammalian body, the total number of nuclear mt genes with T-DMRs will be higher. The differences in protein composition of mitochondria are reported to reflect tissue-dependent nuclear mt gene expression [37]. Our data suggest that DNA methylation status of nuclear mt genes underlies tissue-dependent mitochondrial functions.

\section{Methods}

Mice

C57BL/6N male mice were obtained from Charles River Japan. Mice were euthanized at 12-13 week old, and tissues were collected and frozen at $-80^{\circ} \mathrm{C}$ until use. All experiments using mice were carried out according to the institutional guidelines for the care and use of laboratory animals (Graduate School of Agriculture and Life Sciences, The University of Tokyo).

\section{Genomic DNA extraction}

Genomic DNA was extracted as described previously [11]. Briefly, tissue samples were homogenized, and incubated with lysis solution $(10 \mathrm{mM}$ Tris- $\mathrm{HCl}$ at $\mathrm{pH}$ 8.0, $5 \mathrm{mM}$ EDTA, $200 \mathrm{mM} \mathrm{NaCl}, 0.2 \%$ SDS, and $200 \mu \mathrm{g} / \mathrm{mL}$ proteinase $\mathrm{K}$ ) at $55^{\circ} \mathrm{C}$ for $30 \mathrm{~min}$, and were 


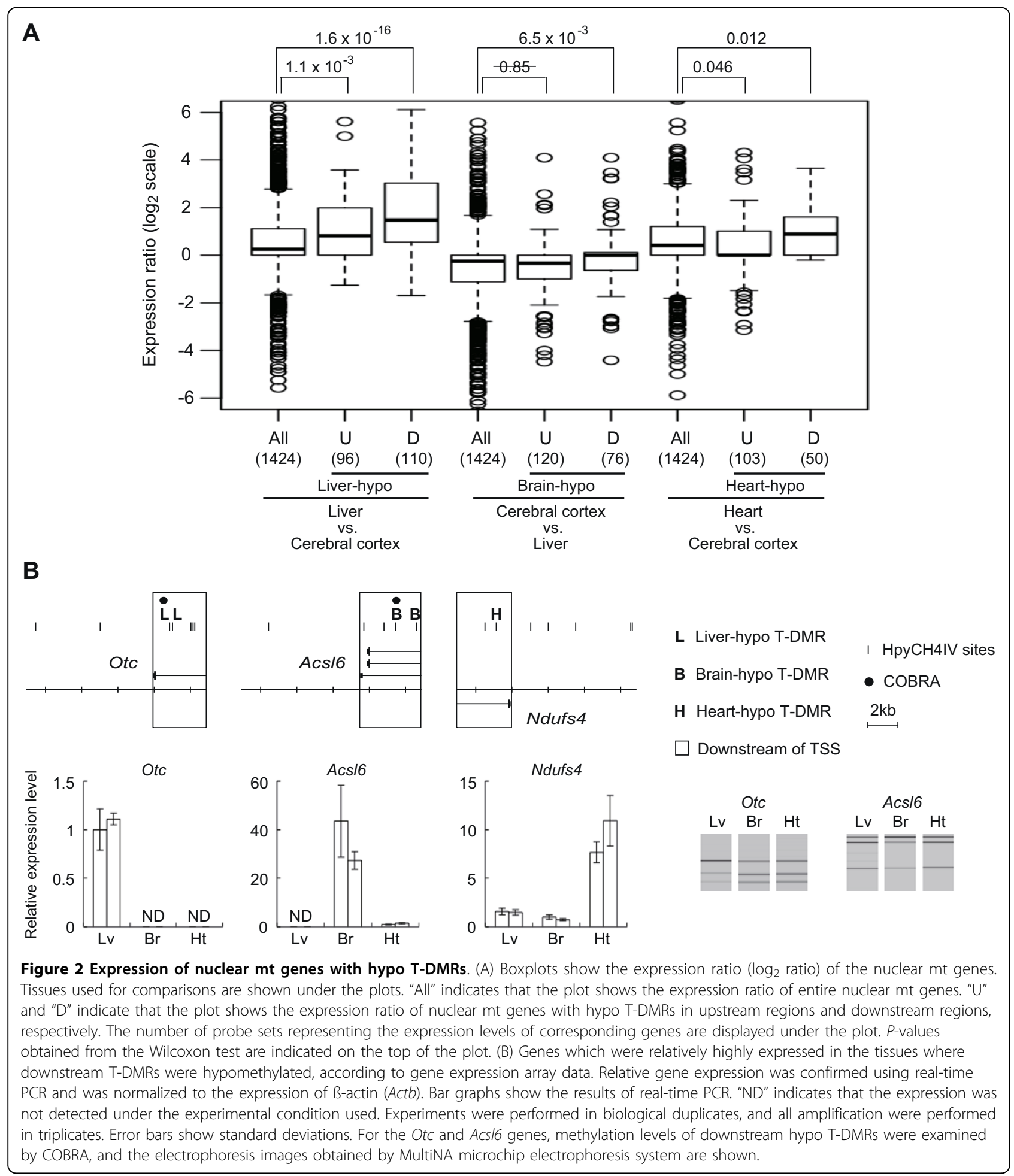

extracted with a phenol/chloroform/isoamylalcohol (PCI) mixture $(50: 49: 1)$, incubated with RNase for 30 min, and re-extracted with PCI. DNA was precipitated with ethanol and dissolved in Tris-EDTA (TE) buffer (pH 8.0).

\section{D-REAM analysis}

We used T-DMR profiling with restriction-tag mediated amplification (D-REAM) analysis [11] to obtain tissuedependent and differentially methylated HpyCH4IV sites within the TSS flanking regions of RefSeq genes. For 


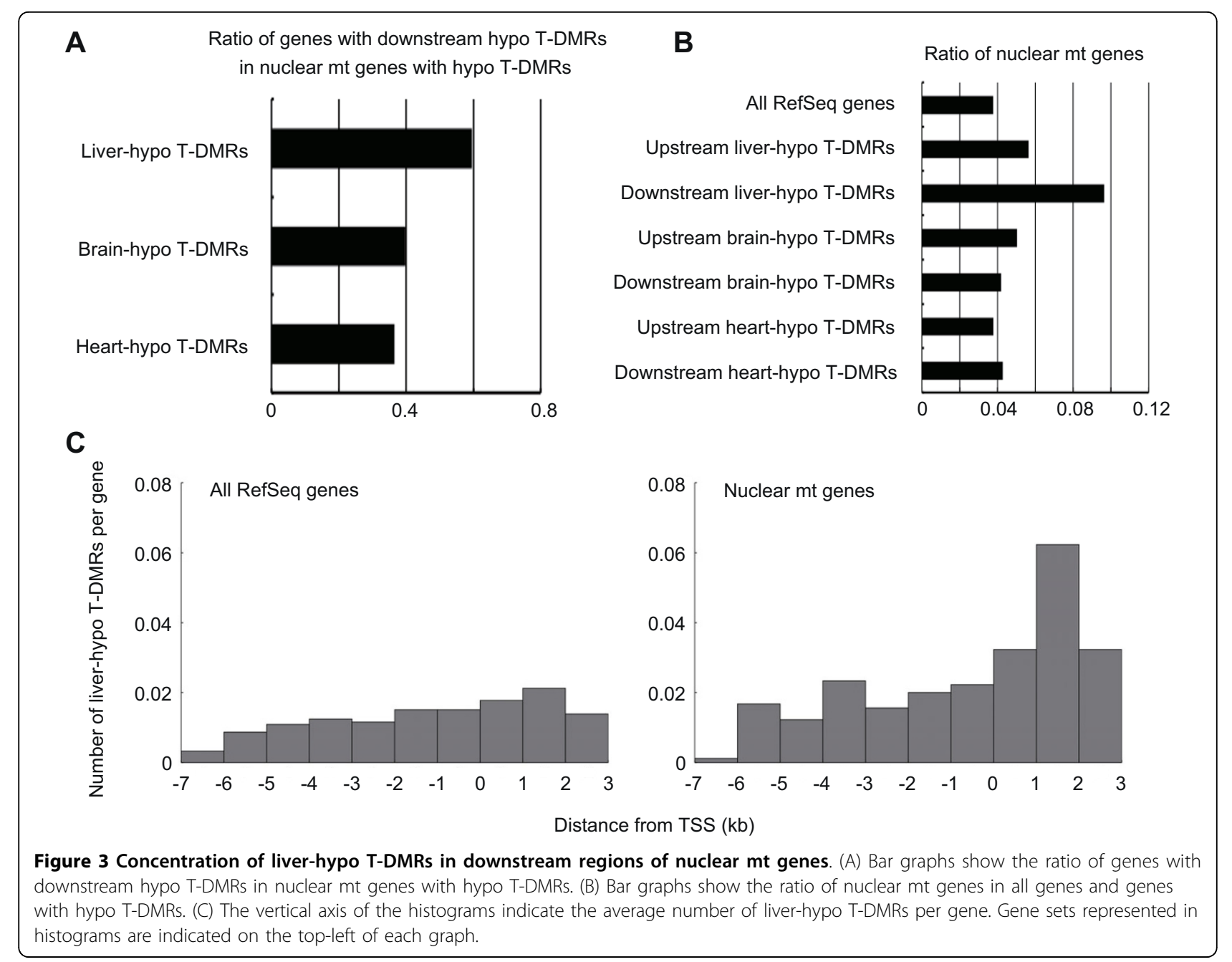

D-REAM analysis, HpyCH4IV-digested genomic DNA was extracted with PCI, re-extracted with chloroform, precipitated with ethanol and dissolved in TE ( $\mathrm{pH} 8.0)$. Using purified DNA (250 ng), following procedure of DREAM analysis was performed as described previously [11]. Briefly, genomic DNA was digested by the methylsensitive enzyme HpyCH4IV (New England Biolabs), followed by ligation-mediated PCR, and subsequent hybridization of DNA to a GeneChip Mouse Promoter 1.0R Array (Affymetrix). Comparison of the resulting signals from digested HpyCH4IV sites between different tissue samples indicates the differential methylation level at a given site.

D-REAM analysis was performed twice for each of the biological duplicates of heart, and once for the liver and brain in this study. For the liver and brain, we added single D-REAM data set from our previous study using tissues from different individual [11]. Correlation coefficients of microarray probe intensities between biological duplicates were greater than 0.93. D-REAM data obtained in this study has been deposited in the ArrayExpress database (accession number A-MEXP-791).

Table 2 Transcription factors whose binding-sites were enriched in analyzed sequences

\begin{tabular}{cccccc}
\hline Gene set & Analyzed sequences & Transcription factor & Number of targets & Enrichment & $\boldsymbol{P}$ value \\
\hline Liver-hypo & 5 kb-downstream & FOXA2 & 52 & 1.23 & $3.1 \times 10^{-2}$ \\
& & NFYA & 29 & 59 & 1.44 \\
Brain-hypo & 5 kb-upstream & CEBPA & 25 & 1.21 & $2.3 \times 10^{-2}$ \\
& & STAT1 & 2.52 & $2.5 \times 10^{-2}$ \\
\hline
\end{tabular}

${ }^{*} P$ values represent results of Fisher's exact test. 
Combined bisulfite restriction analysis (COBRA)

Genomic DNA was digested with HindIII (Takara). Digested DNA $(5 \mu \mathrm{g})$ was denatured with $0.3 \mathrm{M} \mathrm{NaOH}$. Sodium metabisulfite ( $\mathrm{pH}$ 5.0) and hydroquinone were added to a final concentration of $2.0 \mathrm{M}$ and $0.5 \mathrm{mM}$, respectively. The reaction mixture was incubated under following conditions: 15 cycles of $95^{\circ} \mathrm{C}$ for $30 \mathrm{~s}$ and $50^{\circ} \mathrm{C}$ for $15 \mathrm{~min}$. Next, 1.77 volume of QG buffer was added to the reaction mixture, and DNA was purified using a Quiagen gel extraction kit (Qiagen), and eluted with 100 $\mu$ l of elution buffer (EB). DNA was treated with $0.3 \mathrm{M}$ $\mathrm{NaOH}$ at $37^{\circ} \mathrm{C}$ for $15 \mathrm{~min}$, precipitated using $6 \mathrm{M}$ ammonium acetate (pH 7.0) and ethanol, and dissolved in 200 $\mu \mathrm{l}$ TE (pH 8.0). For each bisulfite PCR, $2 \mu \mathrm{l}$ of DNA solution was used as the template, and BIOTAQ HS DNA polymerase (Bioline) was used for amplification. PCR was performed under the following conditions: denaturation at $95^{\circ} \mathrm{C}$ for $10 \mathrm{~min}$ followed by 43 cycles, each cycle comprising $95^{\circ} \mathrm{C}$ for $30 \mathrm{sec}, 60^{\circ} \mathrm{C}$ for $45 \mathrm{sec}, 72^{\circ} \mathrm{C}$ for $30 \mathrm{sec}$, followed by $10 \mathrm{~min}$ at $72^{\circ} \mathrm{C}$. All primers used in this experiment are listed in Additional file 2. The PCR product was digested with HpyCH4IV. Restriction-enzymetreated DNA was desalted using gel filtration through Sephadex G-50, and was analyzed using the MultiNA microchip electrophoresis system (Shimadzu). The methylation level was calculated as the ratio of the amounts of cut fragments to those of the total of cut and uncut fragments obtained from the electropherograms.

\section{RNA extraction, reverse transcription, and real-time PCR}

Total RNA was prepared using TRIzol reagent (Invitrogen). Before synthesis of first-strand cDNA, the RNA preparation was treated with RNase-free DNase I (Invitrogen) to eliminate any residual genomic DNA. The total RNA was then converted into first-strand cDNA using random hexamers and Superscript III First-Strand Synthesis System for RT-PCR (Invitrogen). The obtained cDNA were amplified and quantified in triplicates by using the Quantitect SYBR Green PCR Kit (Qiagen) with ABI 7500 Real Time PCR system (Applied Biosystems). PCR was performed under the following conditions: Incubation at $95^{\circ} \mathrm{C}$ for $10 \mathrm{~min}$ followed by 40 cycles of PCR, each cycle comprising $95^{\circ} \mathrm{C}$ for $15 \mathrm{sec}$ and $60^{\circ} \mathrm{C}$ for $1 \mathrm{~min}$. All primers used in this experiment are listed in Additional file 8. Standard curves were obtained with serial dilutions of a pool of cDNA samples derived from each tissue.

\section{Bioinformatics}

MAT [18] was used to analyze the tiling array data (.CEL files) and identify the hypomethylated regions based on tiling probe signals, probe sequences, and copy numbers. Original tiling probes were remapped to the mouse genome assembly version $\mathrm{mm} 9$ (July 2007 build) provided by UCSC genome database. For the quality control of D-REAM analysis, we monitored the selective amplification of HpyCH4IV-digested fragments for the tilling array data of each sample (Additional file 9).

For expression analysis, data from the GeneChip Mouse Genome 4302.0 Array of liver, heart, and cerebral cortex tissues of C57BL/6N male mice (8-10 week old; $n=2$ for each tissue) were downloaded from Gene Expression Omnibus (accession no. GSE10246). The array image data (.CEL files) was processed by the factor analysis for robust microarray summarization algorithm (FARMS) with quantile normalization [38].

Enrichment analysis of specific transcription factor targets was performed using oPOSSUM program [31]. This program analyzed the genes using one-to-one human-mouse orthologs and detected promoter motifs in the conserved regions. The top $10 \%$ of the non-coding conserved regions with an absolute minimum percent identity of $70 \%$ in each $5 \mathrm{~kb}$ region upstream and downstream of the TSSs were analyzed for vertebrate promoter motifs with a matrix match threshold of $75 \%$.

\section{Additional material}

\section{Additional file 1: Figure S1 Differential methylation levels of HpyCH4IV sites and the corresponding MATscores. \\ Additional file 2: Table S1 Primers used for COBRA, and the result of the experiment. \\ Additional file 3: Table S2 Genomic locations of T-DMRs. \\ Additional file 4: Table S3 Genomic locations of hypo T-DMRs. \\ Additional file 5: Flgure S2 Expression ratio of nuclear $\mathrm{mt}$ genes with hypo T-DMRs. \\ Additional file 6: Table S4 Genes with liver-hypo T-DMRs and FOXA2-binding sites. \\ Additional file 7: Table S5 Genes with hypo T-DMRs and overrepresented transcription factor binding sites. \\ Additional file 8: Table S6 Primers used for real-time PCR. \\ Additional file 9: Figure S3 Distribution of MATscores calculated from D-REAM data of each tissue.}

\section{Acknowledgements}

This research was funded by grants from the National Institute of Biomedical Innovation (NIBIO); Grant-in-Aid for Scientific Research from the Ministry of Education, Culture, Sports, Science, and Technology (MEXT), Japan. The authors declare no conflicts of interest. We would like to thank Dr. Shinya Sato and Mr. Hiroki Muramoto for their helpful suggestions regarding bioinformatic analysis.

\section{Author details}

'Laboratory of Cellular Biochemistry, Department of Animal Resource Science/Veterinary Medical Sciences, the University of Tolyo, Tokyo 113-8657, Japan. ${ }^{2}$ National Institute of Advanced Industrial Science and Technology, Tsukuba, Ibaraki 305-8561, Japan.

\section{Authors' contributions}

MT and KS designed this study. KH performed D-REAM. MT performed COBRA and data analysis with help of SY, and wrote the paper with SY and KS. All authors read and approved the final manuscript. 
Received: 27 February 2010 Accepted: 19 August 2010

Published: 19 August 2010

\section{References}

1. Enns GM: The contribution of mitochondria to common disorders. Mol. Genet. Metab 2003, 80:11-26.

2. Schapira AHV: Mitochondrial disease. Lancet 2006, 368:70-82.

3. Caron F, Jacq C, Rouvière-Yaniv J: Characterization of a histone-like protein extracted from yeast mitochondria. Proc. Natl. Acad. Sci. USA 1979, 76:4265-4269.

4. Dawid IB: 5-methylcytidylic acid: absence from mitochondrial DNA of frogs and HeLa cells. Science 1974, 184:80-81.

5. Groot GS, Kroon AM: Mitochondrial DNA from various organisms does not contain internally methylated cytosine in -CCGG- sequences. Biochim. Biophys. Acta 1979, 564:355-357.

6. Chen T, Li E: Structure and function of eukaryotic DNA methyltransferases. Curr. Top. Dev. Biol 2004, 60:55-89.

7. Klose RJ, Bird AP: Genomic DNA methylation: the mark and its mediators. Trends Biochem. Sci 2006, 31:89-97.

8. Shiota K: DNA methylation profiles of $\mathrm{CpG}$ islands for cellular differentiation and development in mammals. Cytogenet Genome Res 2004, 105:325-334

9. Lieb JD, Beck S, Bulyk ML, Farnham P, Hattori N, Henikoff S, Liu XS, Okumura K, Shiota K, Ushijima T, Greally JM: Applying whole-genome studies of epigenetic regulation to study human disease. Cytogenet Genome Res 2006, 114:1-15.

10. Rakyan VK, Down TA, Thorne NP, Flicek P, Kulesha E, Gräf S, Tomazou EM, Bäckdahl L, Johnson N, Herberth M, Howe KL, Jackson DK, Miretti MM, Fiegler H, Marioni JC, Birney E, Hubbard TJP, Carter NP, Tavaré S, Beck S: An integrated resource for genome-wide identification and analysis of human tissue-specific differentially methylated regions (tDMRs). Genome Res 2008, 18:1518-1529.

11. Yagi S, Hirabayashi K, Sato S, Li W, Takahashi Y, Hirakawa T, Wu G, Hattori N, Hattori N, Ohgane J, Tanaka S, Liu XS, Shiota K: DNA methylation profile of tissue-dependent and differentially methylated regions (T-DMRs) in mouse promoter regions demonstrating tissue-specific gene expression. Genome Res 2008, 18:1969-1978.

12. Hattori N, Nishino K, Ko Y, Hattori N, Ohgane J, Tanaka S, Shiota K: Epigenetic control of mouse Oct-4 gene expression in embryonic stem cells and trophoblast stem cells. J. Biol. Chem 2004, 279:17063-17069.

13. Hattori N, Imao Y, Nishino K, Hattori N, Ohgane J, Yagi S, Tanaka S, Shiota K: Epigenetic regulation of Nanog gene in embryonic stem and trophoblast stem cells. Genes Cells 2007, 12:387-396.

14. Shiota K, Kogo Y, Ohgane J, Imamura T, Urano A, Nishino K, Tanaka S, Hattori N: Epigenetic marks by DNA methylation specific to stem, germ and somatic cells in mice. Genes Cells 2002, 7:961-969.

15. Sato S, Yagi S, Arai Y, Hirabayashi K, Hattori N, Iwatani M, Okita K, Ohgane J, Tanaka S, Wakayama T, Yamanaka S, Shiota K: Genome-wide DNA methylation profile of tissue-dependent and differentially methylated regions (T-DMRs) residing in mouse pluripotent stem cells. Genes Cells 2010, 15:607-618.

16. Rodić N, Oka M, Hamazaki T, Murawski MR, Jorgensen M, Maatouk DM, Resnick JL, Li E, Terada N: DNA methylation is required for silencing of ant4, an adenine nucleotide translocase selectively expressed in mouse embryonic stem cells and germ cells. Stem Cells 2005, 23:1314-1323.

17. Suzuki M, Sato S, Arai Y, Shinohara T, Tanaka S, Greally JM, Hattori N, Shiota K: A new class of tissue-specifically methylated regions involving entire CpG islands in the mouse. Genes Cells 2007, 12:1305-1314.

18. Johnson WE, Li W, Meyer CA, Gottardo R, Carroll JS, Brown M, Liu XS: Model-based analysis of tiling-arrays for ChIP-chip. Proc. Natl. Acad. Sci. USA 2006, 103:12457-12462.

19. Elstner $M$, Andreoli $C$, Ahting U, Tetko I, Klopstock T, Meitinger T, Prokisch H: MitoP2: an integrative tool for the analysis of the mitochondrial proteome. Mol. Biotechnol 2008, 40:306-315.

20. Raijman L: Citrulline synthesis in rat tissues and liver content of carbamoyl phosphate and ornithine. Biochem. J 1974, 138:225-232.

21. Cooper MP, Qu L, Rohas LM, Lin J, Yang W, Erdjument-Bromage $H_{\text {, }}$ Tempst $P$, Spiegelman BM: Defects in energy homeostasis in Leigh syndrome French Canadian variant through PGC-1alpha/LRP130 complex. Genes Dev 2006, 20:2996-3009.
22. Petruzzella $V$, Vergari R, Puzziferri I, Boffoli D, Lamantea E, Zeviani M, Papa S: A nonsense mutation in the NDUFS4 gene encoding the $18 \mathrm{kDa}$ (AQDQ) subunit of complex I abolishes assembly and activity of the complex in a patient with Leigh-like syndrome. Hum. Mol. Genet 2001, 10:529-535.

23. Procaccio $V$, Wallace DC: Late-onset Leigh syndrome in a patient with mitochondrial complex I NDUFS8 mutations. Neurology 2004, 62:1899-1901.

24. Yamashita Y, Kumabe T, Cho YY, Watanabe M, Kawagishi J, Yoshimoto T, Fujino T, Kang MJ, Yamamoto TT: Fatty acid induced glioma cell growth is mediated by the acyl-CoA synthetase 5 gene located on chromosome 10q25.1-q25.2, a region frequently deleted in malignant gliomas. Oncogene 2000, 19:5919-5925.

25. Zainelli GM, Dudek NL, Ross CA, Kim S, Muma NA: Mutant huntingtin protein: a substrate for transglutaminase 1, 2, and 3. J. Neuropathol. Exp. Neurol 2005, 64:58-65.

26. Imai S, Kikuchi R, Kusuhara H, Yagi S, Shiota K, Sugiyama Y: Analysis of DNA methylation and histone modification profiles of liver-specific transporters. Mol. Pharmacol 2009, 75:568-576.

27. Kikuchi R, Yagi S, Kusuhara H, Imai S, Sugiyama Y, Shiota K: Genome-wide analysis of epigenetic signatures for kidney-specific transporters. Kidney Int

28. Wolfrum C, Asilmaz E, Luca E, Friedman JM, Stoffel M: Foxa2 regulates lipid metabolism and ketogenesis in the liver during fasting and in diabetes. Nature 2004, 432:1027-1032.

29. Wolfrum C, Stoffel M: Coactivation of Foxa2 through Pgc-1beta promotes liver fatty acid oxidation and triglyceride/VLDL secretion. Cell Metab 2006, 3:99-110.

30. Wederell ED, Bilenky M, Cullum R, Thiessen N, Dagpinar M, Delaney A, Varhol R, Zhao Y, Zeng T, Bernier B, Ingham M, Hirst M, Robertson G, Marra MA, Jones S, Hoodless PA: Global analysis of in vivo Foxa2-binding sites in mouse adult liver using massively parallel sequencing. Nucleic Acids Res 2008, 36:4549-4564.

31. Ho Sui SJ, Mortimer JR, Arenillas DJ, Brumm J, Walsh CJ, Kennedy BP, Wasserman WW: oPOSSUM: identification of over-represented transcription factor binding sites in co-expressed genes. Nucleic Acids Res 2005, 33:3154-3164.

32. Miura $H$, Tomaru $Y$, Nakanishi $M$, Kondo S, Hayashizaki $Y$, Suzuki M: Identification of DNA regions and a set of transcriptional regulatory factors involved in transcriptional regulation of several human liverenriched transcription factor genes. Nucleic Acids Res 2009, 37:778-792.

33. Ménard C, Hein P, Paquin A, Savelson A, Yang XM, Lederfein D, BarnabéHeider F, Mir AA, Sterneck E, Peterson AC, Johnson PF, Vinson C, Miller FD: An essential role for a MEK-C/EBP pathway during growth factorregulated cortical neurogenesis. Neuron 2002, 36:597-610.

34. Wang J, Schreiber RD, Campbell IL: STAT1 deficiency unexpectedly and markedly exacerbates the pathophysiological actions of IFN-alpha in the central nervous system. Proc. Natl. Acad. Sci. USA 2002, 99:16209-16214.

35. Carmona MC, Iglesias R, Obregón M, Darlington GJ, Villarroya F, Giralt M Mitochondrial biogenesis and thyroid status maturation in brown fat require CCAAT/enhancer-binding protein alpha. J. Biol. Chem 2002, 277:21489-21498.

36. Jang J, Lee C: Mitochondrial adenine nucleotide translocator 3 is regulated by IL-4 and IFN-gamma via STAT-dependent pathways. Cell. Immunol 2003, 226:11-19.

37. Mootha VK, Bunkenborg J, Olsen JV, Hierrild M, Wisniewski JR, Stahl E, Bolouri MS, Ray HN, Sihag S, Kamal M, Patterson N, Lander ES, Mann M: Integrated analysis of protein composition, tissue diversity, and gene regulation in mouse mitochondria. Cell 2003, 115:629-640.

38. Hochreiter S, Clevert D, Obermayer K: A new summarization method for Affymetrix probe level data. Bioinformatics 2006, 22:943-949.

doi:10.1186/1471-2164-11-481

Cite this article as: Takasugi et al: DNA methylation status of nuclearencoded mitochondrial genes underlies the tissue-dependent mitochondrial functions. BMC Genomics 2010 11:481. 